\title{
On a 4-Point Sixteenth-Order King Family of Iterative Methods for Solving Nonlinear Equations
}

\author{
Diyashvir Kreetee Rajiv Babajee $^{\mathbf{1}}$ and Rajinder Thukral ${ }^{2}$ \\ ${ }^{1}$ Department of Applied Mathematical Sciences, School of Innovative Technologies and Engineering, \\ University of Technology, Mauritius, La Tour Koenig, Pointe aux Sables, Mauritius \\ ${ }^{2}$ Padé Research Centre, 39 Deanswood Hill, Leeds, West Yorkshire LS17 5JS, UK \\ Correspondence should be addressed to Diyashvir Kreetee Rajiv Babajee, \\ dbabajee@umail.utm.ac.mu
}

Received 23 March 2012; Accepted 14 May 2012

Academic Editor: Songxiao Li

Copyright (c) 2012 D. K. R. Babajee and R. Thukral. This is an open access article distributed under the Creative Commons Attribution License, which permits unrestricted use, distribution, and reproduction in any medium, provided the original work is properly cited.

\begin{abstract}
A one-parameter 4-point sixteenth-order King-type family of iterative methods which satisfy the famous Kung-Traub conjecture is proposed. The convergence of the family is proved, and numerical experiments are carried out to find the best member of the family. In most experiments, the best member was found to be a sixteenth-order Ostrowski-type method.
\end{abstract}

\section{Introduction}

Solutions of nonlinear equations by iterative methods have been of great interest to numerical analysts. One of the popular methods is the classic Newton method (Newton Raphson method). It has quadratic convergence close to the root, that is the number of good digits is roughly doubled at each iteration. Higher order methods which require the second or higher order derivatives can be costly and thus time consuming. Also, the Newton method can suffer from numerical instabilities. It is consequently important to study higher order variants of Newton's method, which require only function and first derivative calculation and are more robust as compared to Newton's method. Such methods are known as multipoint NewtonLike methods in the Traub sense [1]. Multipoint methods without memory are methods that use new information at a number of points. It is an efficient way of generating higher order methods free from second and higher order derivatives. For a survey of these methods, please refer to [2-4]. In this work, we develop a one-parameter 4-point sixteenth-order King-type family of iterative methods, which satisfy the famous Kung-Traub conjecture. We prove the local convergence of the methods and its asymptotic error constant. We test our methods by 
varying the parameter of the family in a suitable interval and obtain the best value of the parameter for the methods with the highest computational order of convergence. We also compare our methods with other optimal sixteenth order methods. Furthermore, we test the family with two more nonlinear functions and find the best member based on the highest number of successful converging points and lowest mean iteration number.

\section{Preliminaries}

Let $x_{n+1}=\psi\left(x_{n}\right)$ define an iterative function (IF).

Definition 2.1 (see [6]). If the sequence $\left\{x_{n}\right\}$ tends to a limit $x^{*}$ in such a way that

$$
\lim _{n \rightarrow \infty} \frac{x_{n+1}-x^{*}}{\left(x_{n}-x^{*}\right)^{p}}=C
$$

for $p \geq 1$, then the order of convergence of the sequence is said to be $p$, and $C$ is known as the asymptotic error constant. If $p=1, p=2$, or $p=3$, the convergence is said to be linear, quadratic, or cubic, respectively.

Letting $e_{n}=x_{n}-x^{*}$, then the relation

$$
e_{n+1}=C e_{n}^{p}+O\left(e_{n}^{p+1}\right)=O\left(e_{n}^{p}\right)
$$

is called the error equation. The value of $p$ is called the order of convergence of the method.

Definition 2.2 (see [6]). The efficiency index is given by

$$
I E=p^{1 / d},
$$

where $d$ is the total number of new function evaluations (the values of $f$ and its derivatives) per iteration.

Kung-Traub Conjecture (see [7])

Let $\psi$ be an IF without memory with $d$ evaluations. Then

$$
p(\psi) \leq p_{\text {opt }}=2^{d-1},
$$

where $p_{\text {opt }}$ is the maximum order.

We use the approximate computational-order of convergence, COC [8] given by

$$
\mathrm{COC} \approx \frac{\log \left\{\left|x_{n+1}-x^{*}\right| /\left|x_{n}-x^{*}\right|\right\}}{\log \left\{\left|x_{n}-x^{*}\right| /\left|x_{n-1}-x^{*}\right|\right\}} .
$$


International Journal of Mathematics and Mathematical Sciences

\section{Developments of the Methods}

The second-order Newton-Raphson method is given by

$$
\psi_{2 \mathrm{ndNR}}(x)=x-\frac{f(x)}{f^{\prime}(x)}
$$

It is an optimal 1-point IF with efficiency index of 1.414.

A one-parameter King family of fourth-order IF [9] is given by

$$
\psi_{4 \text { th FK }}(x)=\psi_{2 \text { nd NR }}(x)-\frac{f\left(\psi_{2 \text { nd NR }}(x)\right)}{f^{\prime}(x)} \frac{1+\beta t_{1}}{1+(\beta-2) t_{1}} .
$$

The members of the family are 2-point I.F.s with efficiency index of 1.587 . The case $\beta=0$ corresponds to the famous Ostrowski method [6].

Several optimal eight-order methods are developed in [10-12]. Recently, Thukral and Petković [13] developed a family of optimal eighth-order King-type IF given by

$$
\psi 8 \text { th FTPK }(x)=\psi_{4 \text { th FK }}(x)-\left(\phi\left(t_{1}\right)+4 t_{2}+t_{3}\right) \frac{f\left(\psi_{4 \text { th FK }}(x)\right)}{f^{\prime}(x)}
$$

where

$$
t_{1}=\frac{f\left(\psi_{2 \mathrm{nd} \mathrm{NR}}(x)\right)}{f(x)}, \quad t_{2}=\frac{f\left(\psi_{4 \mathrm{th} \mathrm{FK}}(x)\right)}{f(x)}, \quad t_{3}=\frac{f\left(\psi_{4 \mathrm{th} \mathrm{FK}}(x)\right)}{f\left(\psi_{2 \mathrm{nd} \mathrm{NR}}(x)\right)},
$$

and $\phi$ is a weight function satisfying

$$
\phi(0)=1, \quad \phi^{\prime}(0)=2, \quad \phi^{\prime \prime}(0)=10-4 \beta, \quad \phi^{\prime \prime \prime}(0)=12 \beta^{2}-72 \beta+72 .
$$

If we choose

$$
\phi\left(t_{1}\right)=\frac{1+\beta t_{1}+(3 / 2) \beta t_{1}^{2}}{1+(\beta-2) t_{1}+((3 / 2) \beta-1) t_{1}^{2}}
$$

satisfying (3.5), we get a family of optimal eighth-order IF given by

$$
\psi_{8 \text { th FK }}(x)=\psi_{4 \text { th FK }}(x)-\left(\sum_{i=0}^{3} \theta_{i}\right) \frac{f\left(\psi_{4 \text { th FK }}(x)\right)}{f^{\prime}(x)},
$$


where

$$
\begin{aligned}
& \theta_{0}=1, \\
& \theta_{1}=\frac{1+\beta t_{1}+(3 / 2) \beta t_{1}^{2}}{1+(\beta-2) t_{1}+((3 / 2) \beta-1) t_{1}^{2}}-1, \\
& \theta_{2}=t_{3}, \\
& \theta_{3}=4 t_{2} .
\end{aligned}
$$

The members of the family are 3-point eighth-order I.F.s with efficiency index of 1.682. Geum and Kim [14] developed a biparametric family of optimally convergent sixteenth-order 4-point I.F. with their fourth-step weighting function as a sum of a rational and a generic two-variable function:

$$
\begin{gathered}
y=x-\frac{f(x)}{f^{\prime}(x)}, \\
z=y-K_{1}\left(u_{1}\right) \frac{f(y)}{f^{\prime}(x)}, \\
s=z-K_{2}\left(u_{1}, u_{2}, u_{3}\right) \frac{f(s)}{f^{\prime}(x)}, \\
\psi_{16 \text { th FGK } 1}(x)=s-K_{3}\left(u_{1}, u_{2}, u_{3}, u_{4}\right) \frac{f(s)}{f^{\prime}(x)},
\end{gathered}
$$

where

$$
\begin{aligned}
K_{1}\left(u_{1}\right) & =\frac{1+\beta u_{1}+(-9+5 \beta / 2) u_{1}^{2}}{1+(\beta-2) u_{1}+(-4+\beta / 2)} \\
K_{2}\left(u_{1}, u_{2}, u_{3}\right) & =\frac{1+2 u_{1}+(2+\sigma) u_{3}}{1-u_{2}+\sigma u_{3}}, \\
K_{3}\left(u_{1}, u_{2}, u_{3}, u_{4}\right) & =\frac{1+2 u_{1}+(2+\sigma) u_{2} u_{3}}{1-u_{2}-2 u_{3}-u_{4}+2(1+\sigma) u_{2} u_{3}}+K_{4}\left(u_{1}, u_{3}\right)
\end{aligned}
$$

are weighting functions, $K_{4}: \mathbb{C}^{2} \rightarrow \mathbb{C}$ is an analytic function in a region containing the region $(0,0), \beta, \sigma$ are to be chosen freely, and

$$
u_{1}=\frac{f(y)}{f(x)}, \quad u_{2}=\frac{f(z)}{f(y)}, \quad u_{3}=\frac{f(z)}{f(x)}, \quad u_{4}=\frac{f(s)}{f(z)}
$$


We consider the case $(\beta, \sigma)=(2,-2)$ and

$$
K_{4}\left(u_{1}, u_{3}\right)=1-6 u_{1}-u_{1}^{2}-24 u_{1}^{3}-\frac{1}{2}\left(-2 u_{1}+2\right) u_{3}^{2}
$$

for numerical experiments and term the I.F. as 16th GK1.

Geum and Kim [15] proposed another family of optimal sixteenth-order 4-point I.F.s with a linear fraction plus a trivariate polynomial as the fourth-step weighting function. Their family is given by

$$
\psi_{16 \text { th FGK2 }}(x)=s-\frac{1+2 u_{1}}{1-u_{2}-2 u_{3}-u_{4}} \frac{f(s)}{f^{\prime}(x)}+K_{5}\left(u_{1}, u_{2}, u_{3}\right)
$$

where $K_{5}: \mathbb{C}^{3} \rightarrow \mathbb{C}$ is an analytic function in a region containing the region $(0,0,0)$ and $\beta, \sigma$ are to be chosen freely.

We consider the case $(\beta, \sigma)=(24 / 11,-2)$ and

$$
K_{5}\left(u_{1}, u_{2}, u_{3}\right)=-6 u_{1}^{3} u_{2}-\frac{244}{11} u_{1}^{4} u_{3}+6 u_{3}^{2}+u_{1}\left(2 u_{2}^{2}+4 u_{2}^{3}+u_{3}-2 u_{2}^{2}\right)
$$

for numerical experiments and term the I.F. as 16th GK2.

We observe that the 16th FGK1 and 16th FGK2 family of IFs require two parameters and an analytic function. Therefore, we develop a simplified one-parameter optimal 4-point sixteenth-order King-type family of IFs based on 4th FK and 8th FK families. We propose the following family:

$$
\psi_{16 \text { th FK }}(x)=\psi_{8 \text { th FK }}(x)-\left(\sum_{i=0}^{7} \theta_{i}\right) \frac{f\left(\psi_{8 \text { th FK }}(x)\right)}{f^{\prime}(x)}
$$

where

$$
\begin{gathered}
\theta_{4}=t_{5}+t_{1} t_{2}, \\
\theta_{5}=2 t_{1} t_{5}+4(1-\beta) t_{1}^{3} t_{3}+2 t_{2} t_{3}, \\
\theta_{6}=2 t_{6}+\left(7 \beta^{2}-\frac{47}{2} \beta+14\right) t_{3} t_{1}^{4}+(2 \beta-3) t_{2}^{2}+(5-2 \beta) t_{5} t_{1}^{2}-t_{3^{\prime}}^{3}, \\
\theta_{7}=8 t_{4}+\left(-12 \beta+12+2 \beta^{2}\right) t_{5} t_{1}^{3}-4 t_{3}^{3} t 1 \\
+\left(-2 \beta^{2}-22+12 \beta\right) t_{3}^{2} t_{1}^{3}+\left(46+\frac{127}{2} \beta^{2}-105 \beta-10 \beta^{3}\right) t_{2} t_{1}^{4}, \\
t_{4}=\frac{f\left(\psi 8^{\text {th }} F K\right.}{f(x)}, \quad t_{5}=\frac{f\left(\psi_{8^{\text {th }} F K}(x)\right)}{f\left(\psi_{4^{\text {th }} F K}(x)\right)}, \quad t_{6}=\frac{f\left(\psi_{8^{\text {th }} F K}(x)\right)}{f\left(\psi_{2^{\text {nd } N R}}(x)\right)} .
\end{gathered}
$$




\section{Convergence Analysis of the 16th FK Family of IFs}

In this section, we prove the local and sixteenth-order of the 16th FK family of I.F.s using classical Taylor expansion.

Theorem 4.1. Let a sufficiently smooth function $f: D \subset \mathbb{R} \rightarrow \mathbb{R}$ have a simple root $x^{*}$ in the open interval $D$. Then the class of methods without memory (3.15) is of local sixteenth-order convergence.

Proof. Let

$$
c_{j}=\frac{f^{(j)\left(x^{*}\right)}}{j ! f^{\prime}\left(x^{*}\right)}, \quad j=2,3,4, \ldots
$$

Using the Taylor series and the symbolic software such as Maple we have

$$
\begin{gathered}
f(x)=f^{\prime}\left(x^{*}\right)\left[e_{n}+c_{2} e_{n}^{2}+c_{3} e_{n}^{3}+c_{4} e_{n}^{4}+\cdots\right], \\
f^{\prime}(x)=f^{\prime}\left(x^{*}\right)\left[1+2 c_{2} e_{n}+3 c_{3} e_{n}^{2}+4 c_{4} e_{n}^{3}+\cdots\right],
\end{gathered}
$$

so that

$$
\begin{gathered}
u(x)=e_{n}-c_{2} e_{n}^{2}+2\left(c_{2}^{2}-c_{3}\right) e_{n}^{3}+\left(7 c_{2} c_{3}-4 c_{2}^{3}-3 c_{4}\right) e_{n}^{4}+\cdots \\
\psi_{2 \text { nd NR }}(x)-x^{*}=c_{2} e_{n}^{2}-2\left(c_{2}^{2}-c_{3}\right) e_{n}^{3}-\left(7 c_{2} c_{3}-4 c_{2}^{3}-3 c_{4}\right) e_{n}^{4}+\cdots
\end{gathered}
$$

Now, the Taylor expansion of $f(y)$ about $x^{*}$ gives

$$
f(y)=f^{\prime}\left(x^{*}\right)\left[\left(y-x^{*}\right)+c_{2}\left(y-x^{*}\right)^{2}+c_{3}\left(y-x^{*}\right)^{3}+c_{4}\left(y-x^{*}\right)^{4}+\cdots\right] .
$$

Using (4.2), (4.6), and (4.5), we have

$$
\begin{aligned}
t_{1}= & c_{2} e_{n}+\left(2 c_{3}-3 c_{2}^{2}\right) e_{n}^{2}+\left(3 c_{4}-10 c_{2} c_{3}+8 c_{2}^{3}\right) e_{n}^{3} \\
& +\left(-14 c_{2} c_{4}+37 c_{3} c_{2}^{2}-20 c_{2}^{4}-8 c_{3}^{2}+4 c_{5}\right) e_{n}^{4}+\cdots,
\end{aligned}
$$

so that

$$
\psi_{4 \mathrm{th} \mathrm{FK}}(x)-x^{*}=\left((1+2 \beta) c_{2}^{3}-c_{2} c_{3}\right) e_{n}^{4}+\cdots
$$


Similarly, we have

$$
\begin{gathered}
t_{2}=\left((1+2 \beta) c_{2}^{3}-c_{2} c_{3}\right) e_{n}^{3} \\
+\left(-2 c_{3}^{2}+(9+12 \beta) c_{2}^{2} c_{3}+\left(-5-14 \beta-2 \beta^{2}\right) c_{2}^{4}-2 c_{2} c_{4}\right) e_{n}^{4}+\cdots \\
t_{3}=\left((1+2 \beta) c_{2}^{3}-c_{2} c_{3}\right) e_{n}^{2}+\left((4+8 \beta) c_{2} c_{3}+\left(-2-8 \beta-2 \beta^{2}\right) c_{2}^{3}-2 c_{4}\right) e_{n}^{3}+\cdots
\end{gathered}
$$

so that

$$
\begin{aligned}
\theta_{0}= & 1 \\
\theta_{1}= & \left((4 \beta+2) c_{2}^{2}-2 c_{3}\right) e_{n}^{2} \\
& +\left((16 \beta+8) c_{2} c_{3}+\left(-16 \beta-4 \beta^{2}-4\right) c_{2}^{3}-4 c_{4}\right) e_{n}^{3}+\cdots, \\
\theta_{2}= & \left((1+2 \beta) c_{2}^{3}-c_{2} c_{3}\right) e_{n}^{2} \\
& +\left((4+8 \beta) c_{2} c_{3}+\left(-2-8 \beta-2 \beta^{2}\right) c_{2}^{3}-2 c_{4}\right) e_{n}^{3}+\cdots, \\
\theta_{3}= & \left((4+8 \beta) c_{2}^{3}-4 c_{2} c_{3}\right) e_{n}^{3}+\cdots,
\end{aligned}
$$

and finally we get

$$
\begin{aligned}
\psi_{8 \mathrm{th} \mathrm{FK}}(x)-x^{*}=( & \left(-c_{3} c_{2}^{2}+(1+2 \beta) c_{2}^{4}\right) c_{4}-c_{2} c_{3}^{3}+(16+4 \beta) c_{2}{ }^{3} c_{3}{ }^{2} \\
& +\left(-27-\frac{111}{2} \beta-\beta^{2}\right) c_{2}^{5} c_{3} \\
& \left.+\left(12+\frac{95}{2} \beta+44 \beta^{2}-6 \beta^{3}\right) c_{2}^{7}\right) e_{n}^{8}+\ldots
\end{aligned}
$$

By a similar argument, we have

$$
\begin{aligned}
t_{4}= & \left(\left(-c_{3} c_{2}^{2}+(1+2 \beta) c_{2}^{4}\right) c_{4}-c_{2} c_{3}^{3}+(16+4 \beta) c_{2}^{3} c_{3}^{2}\right. \\
& \left.+\left(-27-\frac{111}{2} \beta-\beta^{2}\right) c_{2}^{5} c_{3}+\left(12+\frac{95}{2} \beta+44 \beta^{2}-6 \beta^{3}\right) c_{2}^{7}\right) e_{n}^{7}+\cdots \\
t_{5}= & \left(c_{3}^{2}+(-15-2 \beta) c_{2}^{2} c_{3}+\left(12-3 \beta^{2}+\frac{47}{2} \beta\right) c_{2}^{4}+c_{2} c_{4}\right) e_{n}^{4}+\cdots,
\end{aligned}
$$




$$
\begin{aligned}
t_{6}=( & \left(-c_{2} c_{3}+(1+2 \beta) c_{2}^{3}\right) c_{4}-c_{3}^{3} \\
& +(16+4 \beta) c_{2}^{2} c_{3}^{2}+\left(-27-\frac{111}{2} \beta-\beta^{2}\right) c_{2}{ }^{4} c_{3} \\
& \left.+\left(12+\frac{95}{2} \beta+44 \beta^{2}-6 \beta^{3}\right) c_{2}^{6}\right) e_{n}^{6}+\cdots,
\end{aligned}
$$

so that

$$
\begin{aligned}
\theta_{4}= & \left(c_{3}^{2}+(-16-2 \beta) c_{2}^{2} c_{3}+\left(13-3 \beta^{2}+\frac{51}{2} \beta\right) c_{2}^{4}+c_{2} c_{4}\right) e_{n}^{4}+\cdots, \\
\theta_{5}= & \left(4 c_{2} c_{3}^{2}+(-38-8 \beta) c_{2}^{3} c_{3}+\left(30-6 \beta^{2}+59 \beta\right) c_{2}^{5}+2 c_{2}^{2} c_{4}\right) e_{n}^{5}+\cdots, \\
\theta_{6}= & \left(\left(-2 c_{2} c_{3}+(7+2 \beta) c_{2}^{3}\right) c_{4}-c_{3}^{3}+(31+2 \beta) c_{2}^{2} c_{3}^{2}\right. \\
& \left.+\left(-134-\beta^{2}-\frac{95}{2} \beta\right) c_{2}^{4} c_{3}+\left(-30 \beta^{2}+94+8 \beta^{3}+177 \beta\right) c_{2}^{6}\right) e_{n}^{6}+\cdots, \\
\theta_{7}= & \left(\left(-8 c_{3} c_{2}^{2}+\left(20+4 \beta+2 \beta^{2}\right) c_{2}^{4}\right) c_{4}-4 c_{2} c_{3}^{3}+(8 \beta+106) c_{2}^{3} c_{3}^{2}\right. \\
& +\left(-\frac{147}{2} \beta^{2}+14 \beta^{3}-386-71 \beta\right) c_{2}^{5} c_{3} \\
& \left.+\left(-\frac{357}{2} \beta^{2}-34 \beta^{4}+260+405 \beta+160 \beta^{3}\right) c_{2}^{7}\right) e_{n}^{7}+\cdots,
\end{aligned}
$$

and finally we get

$$
\begin{aligned}
\psi_{16 \text { th FK }}( & x)-x^{*} \\
= & \left(\left(-c_{2}^{3} c_{3}^{4}+\left(-c_{3}^{2} c_{2}^{4}+(4 \beta+2) c_{2}^{6} c_{3}+\left(-1-4 \beta-4 \beta^{2}\right) c_{2}^{8}\right) c_{4}\right.\right. \\
& +(6 \beta+17) c_{2}^{5} c_{3}^{3}+\left(-12-82 \beta^{3}-139 \beta^{2}+12 \beta^{4}-\frac{143}{2} \beta\right) c_{2}^{11} \\
& \left.+\left(-9 \beta^{2}-43-\frac{183}{2} \beta\right) c_{2}^{7} c_{3}^{2}+\left(39+156 \beta^{2}+157 \beta-4 \beta^{3}\right) c_{2}^{9} c_{3}\right) c_{5} \\
& +\left(-c_{2}^{4} c_{3}+(1+2 \beta) c_{2}^{6}\right) c_{4}^{3} \\
& +\left((16 \beta+66) c_{2}^{5} c_{3}^{2}+\left(-4 \beta^{4}+81+219 \beta+124 \beta^{2}+18 \beta^{3}\right) c_{2}^{9}\right. \\
& \left.+\left(-189 \beta+2 \beta^{3}-26 \beta^{2}-143\right) c_{2}^{7} c_{3}-4 c_{2}^{3} c_{3}^{3}\right) c_{4}^{2}
\end{aligned}
$$




$$
\begin{aligned}
& +\left((22 \beta+140) c_{2}^{4} c_{3}^{4}+\left(9 \beta^{4}-210 \beta^{3}+\frac{6805}{4} \beta^{2}+3933+4784 \beta\right) c_{2}^{8} c_{3}^{2}\right. \\
& +\left(2 \beta^{3}-699 \beta-1349-34 \beta^{2}\right) c_{2}^{6} c_{3}^{3} \\
& -4 c_{2}^{2} c_{3}^{5}+\left(-\frac{19539}{4} \beta^{2}+891 \beta^{4}-4255-10278 \beta-2682 \beta^{3}-72 \beta^{5}\right) c_{2}^{10} c_{3} \\
& \left.+\left(1535+92 \beta^{6}+\frac{11823}{2} \beta^{2}+5963 \beta+\frac{2829}{2} \beta^{3}+1863 \beta^{4}-882 \beta^{5}\right) c_{2}^{12}\right) c_{4} \\
& +\left(-20627 \beta^{6}+4028 \beta^{7}-240 \beta^{8}+\frac{88033}{2} \beta+\frac{260321}{4} \beta^{2}+\frac{134363}{8} \beta^{3}+\frac{40171}{4} \beta^{4}\right. \\
& \left.+\frac{58839}{2} \beta^{5}+8484\right) c_{2}^{15}-c_{3}^{7} c_{2}+(6 \beta+60) c_{2}^{3} c_{3}^{6}+\left(\beta^{2}-\frac{777}{2} \beta-1134\right) c_{2}^{5} c_{3}^{5} \\
& +\left(-3778 \beta^{6}-32541-\frac{234997}{2} \beta+\frac{60929}{2} \beta^{5}\right. \\
& \left.\quad-60462 \beta^{4}+62 \beta^{7}-95165 \beta^{2}-\frac{75499}{4} \beta^{3}\right) c_{2}^{13} c_{3} \\
& +\left(\frac{12567}{2} \beta-242 \beta^{3}+\frac{4585}{4} \beta^{2}+17 \beta^{4}+8769\right) c_{2}^{7} c_{3}^{4} \\
& +\left(177 \beta^{6}-\frac{133}{2} \beta^{5}-\frac{52923}{4} \beta^{4}+46415+\frac{354947}{8} \beta^{3}+103054 \beta+\frac{167505}{4} \beta^{2}\right) c_{2}^{11} c_{3}^{2} \\
& \left.+\left(-35257 \beta-30052-108 \beta^{5}-\frac{74875}{4} \beta^{2}+\frac{725}{2} \beta^{3}+920 \beta^{4}\right) c_{2}^{9} c_{3}^{3}\right) e_{n}^{16}+\ldots \\
& +
\end{aligned}
$$

In the next section, we carry out numerical experiments to find the best member of the family and compare it to the Geum and Kim sixteenth-order IFs

\section{Numerical Experiments}

The test functions and their exact root $x^{*}$ are displayed in Table 1. The approximation $x_{n}$ is calculated by using the same total number of function evaluations (TNFE) for all I.F.s considered. In the calculations, 15 TNFE are used by each I.F. For the 16th FK family, we choose a suitable range of values of $\beta$, which are based on the initial approximation of the root. ${ }^{a}$ indicates the values of $\beta$ excluded in the range because of invalid estimate. The best value of $\beta$ is chosen based on the smallest value of $\left|x_{3}-x^{*}\right|$ and the highest computational-order of convergence (COC). The range and best value of $\beta$ are given in Table 2 for each function with its starting point. For most functions, the best value of $\beta=0$, which corresponds to optimal 4-point sixteenth-order Ostrowski-type I.F. Furthermore, the approximation $x_{3}-x^{*}$ and the computational order of convergence (COC) for the best member of the 16th FK family and the 16th GK1 and 16th GK2 IFs are displayed in Table 3. The results show that the best member of the 16th FK family gives the smallest value of $\left|x_{3}-x^{*}\right|$ for $f_{4}, f_{6}, f_{7}$ when compared to Geum and Kim sixteenth order IF. 
Table 1: Test functions and their roots.

\begin{tabular}{lr}
\hline$f_{1}(x)=\exp (x) \sin (x)+\ln \left(1+x^{2}\right)$ & $x^{*}=0$ \\
$f_{2}(x)=x^{15}+x^{4}+4 x^{2}-15$ & $x^{*}=1.148538 \ldots$ \\
$f_{3}(x)=(x-2)\left(x^{10}+x+1\right) \exp (-x-1)$ & $x^{*}=2$ \\
$f_{4}(x)=(x+1) \exp (\sin (x))-x^{2} \exp (\cos (x))-1$ & $x^{*}=0$ \\
$f_{5}(x)=\sin ^{2}(x)-x^{2}+1$ & $x^{*}=1.40449165 \ldots$ \\
$f_{6}(x)=\exp (-x)-\cos (x)$ & $x^{*}=0$ \\
$f_{7}(x)=\ln \left(x^{2}+x+2\right)-x+1$ & $x^{*}=4.15259074 \ldots$ \\
\hline
\end{tabular}

Table 2: Range and the best value of $\beta$ for the 16th FK family.

\begin{tabular}{lcc}
\hline Function & Range & Best value of $\beta$ \\
\hline$f_{1}, x_{0}=1$ & {$[-5,5]^{\mathrm{a}}, a=-3,-2$} & 0 \\
$f_{2}, x_{0}=1.3$ & {$[-5,5]^{\mathrm{b}}, b=-3,-2,-1$} & 0 \\
$f_{3}, x_{0}=2.5$ & {$[-5,5]^{\mathrm{c}}, c=-1$} & 0 \\
$f_{4}, x_{0}=0.25$ & {$[-5,5]$} & 1 \\
$f_{5}, x_{0}=2.5$ & {$[-2,5]$} & 0 \\
$f_{6}, x_{0}=1 / 6$ & {$[-5,4]$} & 0 \\
$f_{7}, x_{0}=3.5$ & {$[-5,5]$} & -1 \\
\hline
\end{tabular}

Table 3: Comparison of optimal 4-point sixteenth-order I.F.s.

\begin{tabular}{lcccccc}
\hline \multirow{2}{*}{ function } & \multicolumn{2}{c}{16 th FK } & \multicolumn{2}{c}{16 th GK1 } & \multicolumn{2}{c}{ 16th GK2 } \\
& $\left|x_{3}-x^{*}\right|$ & COC & $\left|x_{3}-x^{*}\right|$ & COC & $\left|x_{3}-x^{*}\right|$ & COC \\
\hline$f_{-1}, x_{-} 0=1$ & $0.137 \mathrm{e}-362$ & 15.989 & $0.353 \mathrm{e}-581$ & 15.981 & $0.103 \mathrm{e}-522$ & 15.998 \\
$f \_2, x_{-} 0=1.3$ & $0.898 \mathrm{e}-670$ & 16.000 & $0.994 \mathrm{e}-782$ & 16.000 & $0.723 \mathrm{e}-811$ & 15.987 \\
$f \_3, x_{-} 0=2.5$ & $0.479 \mathrm{e}-200$ & 15.928 & fail & fail & $0.819 \mathrm{e}-316$ & 15.978 \\
$f \_4, x_{-} 0=0.25$ & $0.492 \mathrm{e}-3155$ & 16.000 & $0.241 \mathrm{e}-2455$ & 16.000 & $0.516 \mathrm{e}-2071$ & 16.000 \\
$f \_5, x_{-} 0=2.5$ & $0.142 \mathrm{e}-810$ & 16.000 & $0.164 \mathrm{e}-1147$ & 15.993 & $0.452 \mathrm{e}-1020$ & 16.000 \\
$f \_6, x_{-} 0=1 / 6$ & $0.224 \mathrm{e}-1702$ & 16.000 & $0.172 \mathrm{e}-1004$ & 15.992 & $0.782 \mathrm{e}-892$ & 16.000 \\
$f_{-7,} x_{-} 0=3.5$ & $0.927 \mathrm{e}-4464$ & 16.000 & $0.241 \mathrm{e}-4144$ & 16.000 & $0.136 \mathrm{e}-3763$ & 16.000 \\
\hline
\end{tabular}

We next test the 16th FK family by varying the starting points. Let us consider the functions $f_{2}$ and $f_{7}$. We focus on the behaviour of the IFs with the starting points, which are equally spaced with $\Delta x=0.1$ in the intervals $(-3.9,6.1]$ for $f_{2}$ and $(-0.9,9.1]$ for $f_{7}$ to check the robustness of the IFs. A starting point was considered as divergent if it does not satisfy the condition $\left|x_{n+1}-x_{n}\right|<10^{-13}$ in at most 100 iterations. We denote the quantity $\omega_{c}$ as the mean number of iterations from a successful starting point until convergence with $\left|x_{n+1}-x_{n}\right|<10^{-13}$. Let $N_{s}$ denote the number of successful points of 100 starting points. We test for $101 \beta$ of the family with $\Delta \beta=0.1$ in the interval $[-5,5]$. Figure 1 shows the variation of the converging points and mean iteration number with respect to $\beta$ for the function $f_{2}$. We can observe the family is globally convergent for the values of $\beta \in[-1.2,-0.7]$ and $\beta=-0.3,0.1,0.5,0.9$. It is the member $\beta=-0.8$ that has the smallest mean iteration number and is the most efficient member for $f_{2}$. In Figure 2, we observe that the family is globally convergent for the function $f_{7}$ for all given values of $\beta$. It is the member $\beta=0.2$ which has the lowest mean iteration number. Figure 3 shows the number of iterations needed to achieve convergence is 2 for any starting point in the interval [2,8.3] enclosing the root. This illustrates the high speed of convergence of the method. That is, higher order I.F. can converge in few iterations even if 


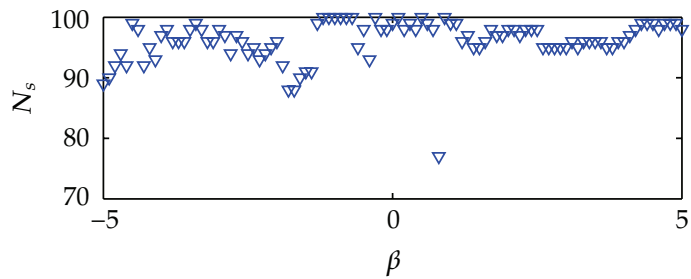

(a)

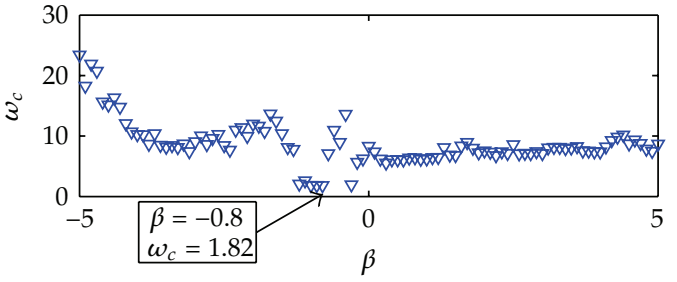

(b)

Figure 1: Behaviour of 16th FK family for the function $f_{2}$.

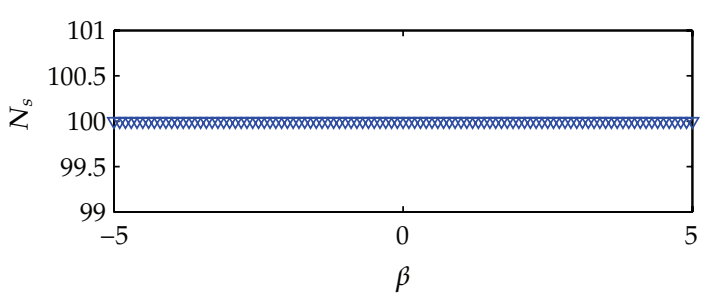

(a)

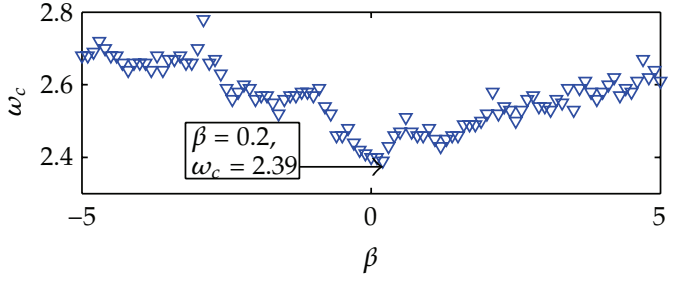

(b)

Figure 2: Behaviour of 16th FK family for the function $f_{7}$.

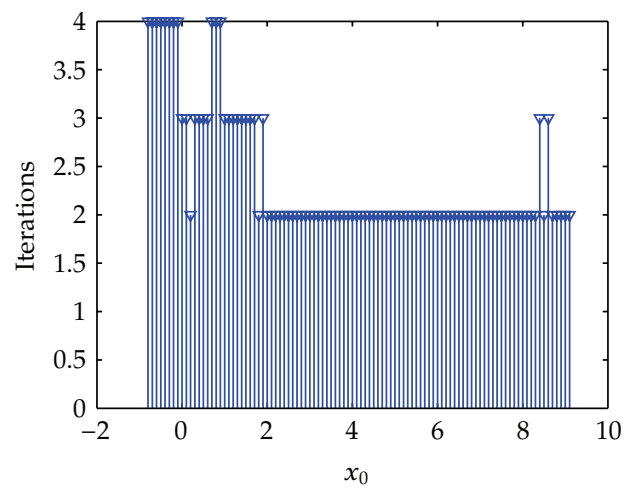

Figure 3: Behaviour of the number of iterations for convergence with the starting point $x_{0}$ of the member $\beta=0.2$ of the 16th FK family for the function $f_{7}$.

the starting point is not very close to the root. We consider two more test functions, one of which is of simple cubic type $[16,17]$

$$
f_{8}(x)=x^{3}+\ln x, \quad x>0, x \in \mathbb{R}
$$

for which the logarithm restricts the function to be positive and its convex properties of the function are favorable for global convergence [16, 17]. We test for 100 starting points in the interval $(0,10]$. The root $x^{*}=0.704709490254913$ correct to 14 digits. A starting point was considered as divergent if it does not satisfy the convergence condition in at most 100 


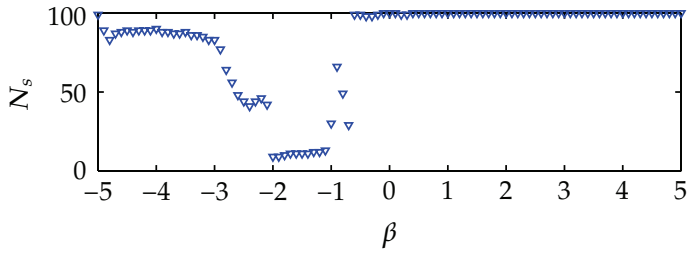

(a)

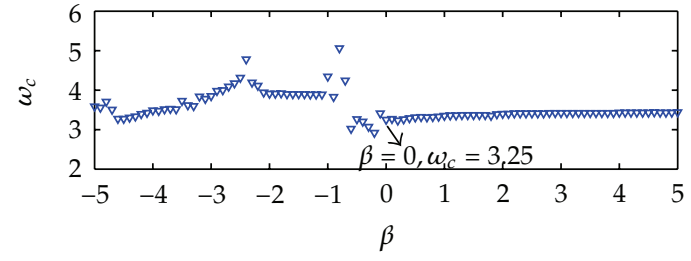

(b)

Figure 4: Behaviour of 16th FK family for the Cubic function.

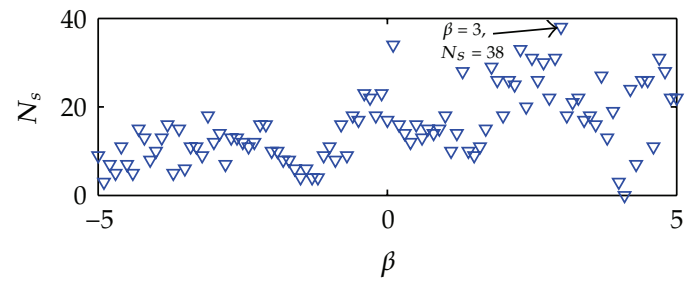

(a)

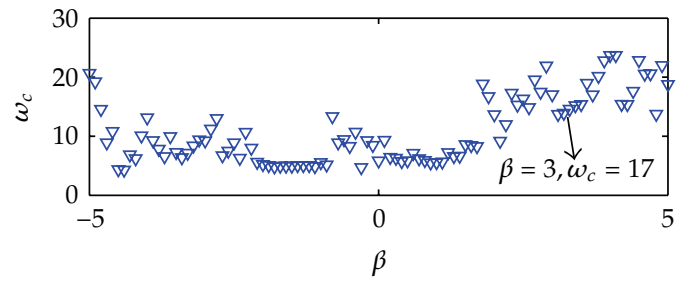

(b)

Figure 5: Behaviour of 16th FK family for the Oscillatory Cubic function.

iterations together with $x \leq 0$ at any iterates. A similar analysis is performed for another test function, the Oscillatory Cubic $[16,17]$ :

$$
f_{9}(x)=x^{3}+\ln x+0.15 \cos (50 x)
$$

in which the single root has been moved marginally to $x^{*}=0.717519716444759$ but many local extrema have been introduced on a small scale [17]. This means that when the iterates of the I.F.s fall in the region where $f^{\prime}(x)=0$, they become zero or negative, causing them to diverge.

Figure 4 shows the variation of the converging points and mean iteration number with respect to $\beta$ for the Cubic function. It can be observed that the 16th FK family is globally convergent for $\beta=-5,-0.1,0,0.1$, and $\beta \geq 0.4$. This is the member $\beta=0$, which is the most efficient I.F. since it is globally convergent with the smallest mean iteration number of 3.25. We note that the family has many diverging points for negative values of $\beta$.

Figure 5 shows the variation of the converging points and mean iteration number with respect to $\beta$ for the Oscillatory Cubic function. It can be observed that due to the perturbations the 16th FK family has difficulty with this function because its members have less than $40 \%$ of starting points successfully converging. The mean of the IFs has also risen. The most efficient member of the family with the highest number of converging points (38) is the member $\beta=3$ with mean 17.

\section{Conclusion}

We develop a 4-point sixteenth order King family of iterative methods. We prove the local convergence of the methods. We test the family via some numerical experiments to find the best member, which corresponds to a sixteenth-order Ostrowski method for most cases. 


\section{Acknowledgments}

The authors are grateful to the unknown referees for their valuable comments to improve the paper. D. K. R. Babajee is an IEEE member.

\section{References}

[1] J. F. Traub, Iterative Methods for the Solution of Equations, Prentice-Hall, New Jersey, NJ, USA, 1964.

[2] D. K. R. Babajee, Analysis of Higher Order Variants of Newton's Method and Their Applications to Differential and Integral Equations and in Ocean Acidification [Ph.D. thesis], University of Mauritius, 2010.

[3] J. M. McNamee, Numerical Methods for Roots of Polynomial: Part 1, Elsevier, Amsterdam, The Netherlands, 2007.

[4] M. S. Petković and L. D. Petković, "Families of optimal multipoint methods for solving nonlinear equations: a survey," Applicable Analysis and Discrete Mathematics, vol. 4, no. 1, pp. 1-22, 2010.

[5] R. Wait, The Numerical Solution of Algebraic Equations, John Wiley \& Sons, New York, NY, USA, 1979.

[6] A. M. Ostrowski, Solutions of Equations and System of Equations, Academic Press, New York, NY, USA, 1960.

[7] H. T. Kung and J. F. Traub, "Optimal order of one-point and multipoint iteration," Journal of the Association for Computing Machinery, vol. 21, no. 4, pp. 643-651, 1974.

[8] S. Weerakoon and T. G. I. Fernando, "A variant of newton's method with accelerated third-order convergence," Applied Mathematics Letters, vol. 13, no. 8, pp. 87-93, 2000.

[9] R. F. King, "A family of fourth order methods for nonlinear equations," SIAM Journal on Numerical Analysis, vol. 10, pp. 876-879, 1973.

[10] W. Bi, H. Ren, and Q. Wu, "Three-step iterative methods with eighth-order convergence for solving nonlinear equations," Journal of Computational and Applied Mathematics, vol. 225, no. 1, pp. 105-112, 2009.

[11] W. Bi, Q. Wu, and H. Ren, "A new family of eighth-order iterative methods for solving nonlinear equations," Applied Mathematics and Computation, vol. 214, no. 1, pp. 236-245, 2009.

[12] B. Neta, "On a family of multipoint methods for nonlinear equations," International Journal of Computer Mathematics, vol. 9, no. 4, pp. 353-361, 1981.

[13] R. Thukral and M. S. Petković, "A family of three-point methods of optimal order for solving nonlinear equations," Journal of Computational and Applied Mathematics, vol. 233, no. 9, pp. 2278-2284, 2010.

[14] Y. H. Geum and Y. I. Kim, "A biparametric family of optimally convergent sixteenth-order multipoint methods with their fourth-step weighting function as a sum of a rational and a generic two-variable function," Journal of Computational and Applied Mathematics, vol. 235, no. 10, pp. 3178-3188, 2011.

[15] Y. H. Geum and Y. I. Kim, "A family of optimal sixteenth-order multipoint methods with a linear fraction plus a trivariate polynomial as the fourth-step weighting function," Computers and Mathematics with Applications, vol. 61, no. 11, pp. 3278-3287, 2011.

[16] D. K. R. Babajee and M. Z. Dauhoo, "An analysis of the properties of the variants of newton's method with third order convergence," Applied Mathematics and Computation, vol. 183, no. 1, pp. 659-684, 2006.

[17] M. Drexler, Newton's Method as a Global Solver for Non-Linear Problems [Ph.D. thesis], University of Oxford, Oxford, UK, 1997. 


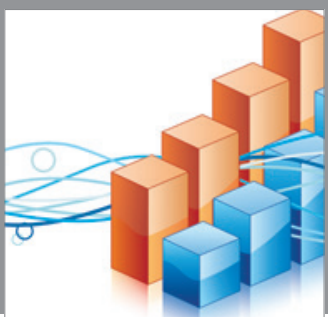

Advances in

Operations Research

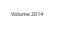

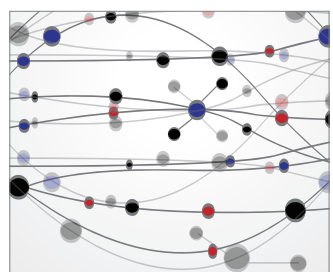

\section{The Scientific} World Journal
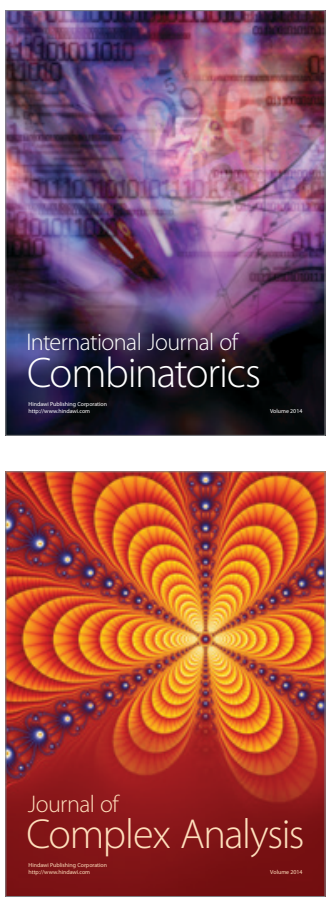

International Journal of

Mathematics and

Mathematical

Sciences
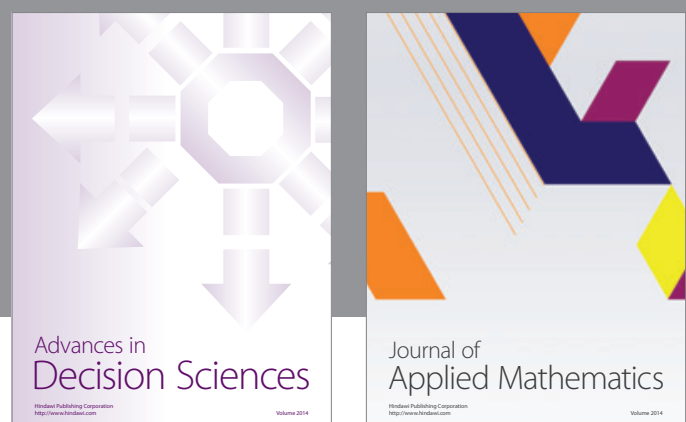

Journal of

Applied Mathematics
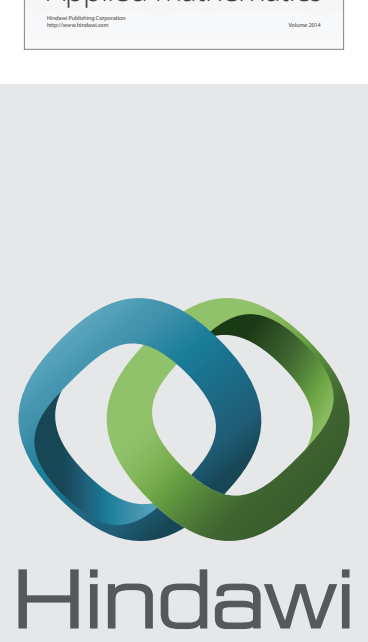

Submit your manuscripts at http://www.hindawi.com
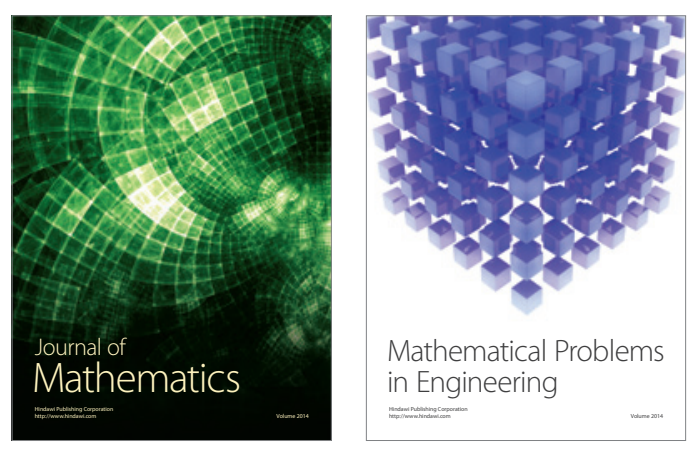

Mathematical Problems in Engineering
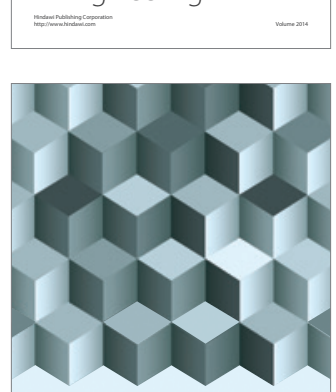

Journal of

Function Spaces
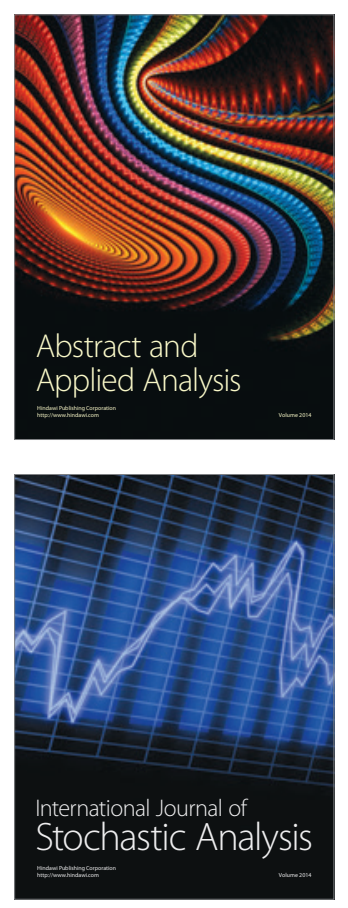

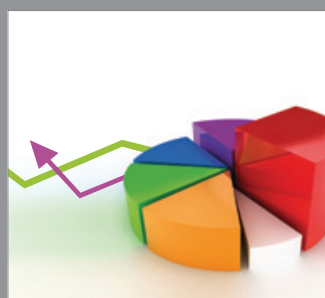

ournal of

Probability and Statistics

Promensencen
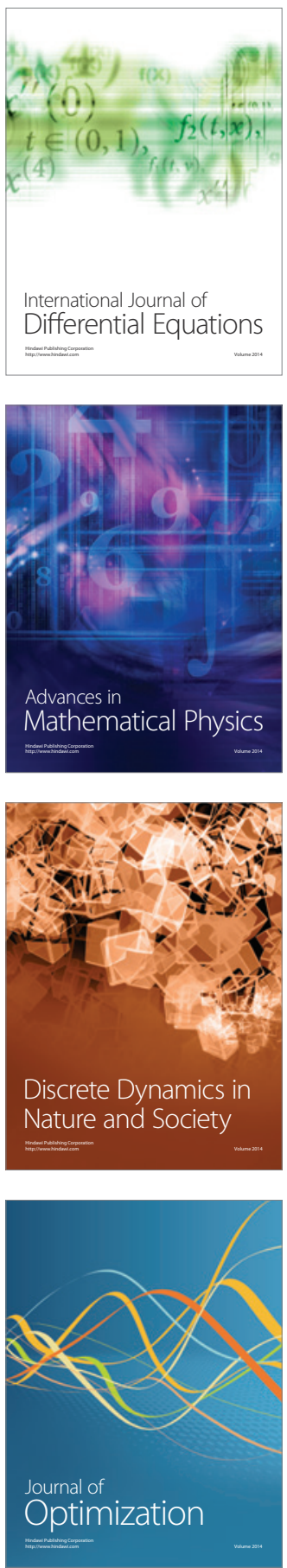